\title{
Distribuição espacial dos casos novos de tuberculose em Vitória, Estado do Espírito Santo, no período entre 2000 e 2005
}

\author{
Spatial distribution of new tuberculosis cases in Vitória, \\ State of Espírito Santo, between 2000 and 2005 \\ Rafael da Cruz Araújo Vieira', Thiago Nascimento do Prado, \\ Maria Guimarães Siqueira ${ }^{1}$, Reynaldo Dietze $^{1}$ e Ethel Leonor Noia Maciel $^{1}$
}

\begin{abstract}
RESUMO
Realizou-se um estudo ecológico com a análise da distribuição espacial dos 979 novos casos de tuberculose entre 2000 e 2005. Utilizou-se o Método Bayesiano Empírico Local para estimação do risco. 0 Índice de Moram Local foi calculado para avaliação das autocorrelações entre as incidências de bairros limítrofes. Observou-se que a tuberculose distribui-se heterogeneamente entre os bairros, sendo possível identificar regiões com alto risco de adoecimento.
\end{abstract}

Palavras-chaves: Tuberculose. Prevenção. Distribuição espacial. Vigilância epidemiológica.

\begin{abstract}
We carried out an ecological study in which the spatial distribution of 979 new tuberculosis cases between 2000 and 2005 were analyzed. The risk was estimated using the local empirical Bayesian method. The local indicators of spatial association were calculated to evaluate autocorrelations of incidence between adjoining districts. We found that the tuberculosis cases were heterogeneously distributed between districts, and it was possible to identify regions in which there was a high risk of becoming ill.
\end{abstract}

Key-words: Tuberculosis. Prevention. Spatial distribution. Epidemiological surveillance.

A tuberculose (TB) é a principal causa de morte por doenças infecciosas entre adultos ao redor do mundo. Para o Brasil, são esperados o surgimento anual de 110 mil casos novos de TB, o que o coloca na $16^{\circ}$ posição entre os países com maior carga de doença ${ }^{17}$.

No Espírito Santo, a endemia apresenta uma incidência de 39,4 casos novos para cada 100 mil habitantes, tendo oito de seus municípios considerados prioritários para o Programa Nacional de Controle da Tuberculose (PNCT), dentre os quais o município de Vitória ${ }^{9}$. Por sua posição central no contexto político-econômico estadual, Vitória convive com grandes desigualdades sociais, o advento da AIDS e o envelhecimento populacional ${ }^{13}$.

Entendendo que o controle efetivo da endemia da TB fundamentam-se na interrupção da sua cadeia de transmissão na comunidade, os programas de controle da doença vêm atuando segundo duas perspectivas: a da atenção individualizada aos pacientes e seus contactantes e a vigilância em saúde dos agrupamentos populacionais ${ }^{5}$. Nesta segunda perspectiva do controle, tem-se buscado compreender a ocorrência da doença num nível ampliado, onde indivíduos e espaço social interagem na produção de locais que propiciam diferenciados riscos de adoecimento, alterando substancialmente a programação de suas ações bem como a sua operacionalizaçã $0^{4}$.

Recentemente, um estudo realizado na Cidade de Recife, PE, utilizou técnicas de geoprocessamento para definir, entre as variáveis estudadas, aquelas caracterizadoras de situações de risco que atuavam sobre determinados locais e capazes de definir a sua distribuição espacial. Neste mesmo trabalho, demonstrouse a necessidade de produção de dados básicos e consistentes a cerca dos casos da doença tanto para caracterizar a distribuição

\footnotetext{
1. Laboratório de Epidemiologia, Núcleo de Doenças Infecciosas, Centro de Ciências da Saúde, Universidade Federal do Espírito Santo, Vitória, ES. Projeto financiado pelo Fundo de Apoio à Ciência e Tecnologia da Prefeitura Municipal de Vitória. Processo 544.9654/2005.

Endereço para correspondência: Dr. Rafael da Cruz Araújo Vieira. Laboratório de Epidemiologia/Núcleo de Doenças Infecciosas/CCS/UFES. Av. Marechal Campos 1648, Maruípe, 29043-091 Vitória, ES.

Tel: 5527 2122-7205; Fax: 5527 3335-7139

e-mail: rcavieira@ndi.ufes.br

Recebido para publicação em: 21/05/2007

Aceito em: 09/11/2007
} 
espacial como para a construção desse novo modelo de vigilância em saúde baseado no território ${ }^{16}$.

Assim, esse estudo teve como objetivo analisar a distribuição espacial do surgimento de casos novos de TB e a detecção de áreas de maior risco de adoecimento no município de Vitória.

Este é um estudo ecológico com análise espacial dos casos novos de TB no período de 2000 a 2005, residentes em Vitória, ES.

Tomou-se como unidade de análise os bairros do município e a base cartográfica foi obtida junto ao Geo-Bases, através da Secretaria de Fazenda da Prefeitura Municipal de Vitória. Os dados populacionais foram adquiridos junto à Fundação Instituto Brasileiro de Geografia e Estatística (IBGE) e os dados epidemiológicos extraídos do Sistema Nacional de Agravos de Notificação (Secretaria de Vigilância em Saúde/Ministério da Saúde), gerenciado pela Secretaria Estadual de Saúde (SESA/ES). Todos os dados da Ficha de Notificação estavam disponíveis individualmente. Por questão de confidencialidade, o acesso a este foi autorizado pela Secretaria Municipal de Saúde e o estudo aprovado pelo Comitê de Ética em Pesquisa do Centro de Ciências da Saúde da UFES, de acordo com o que é estabelecido pela Resolução no 196 (CONEP, de 10 de outubro de 1996), sob o registro $050 / 05$.

As taxas de incidência foram calculadas seguindo a metodologia utilizada pela SVS/MS ${ }^{8}$, utilizando-se o somatório dos casos novos por bairro e a sua população no ano de 2000. A tabulação dos dados do SINAN e o cálculo das taxas de incidência bruta foram feitos na planilha Microsoft Excel.

Foi implementado o método Bayesiano Empírico Local (LEBayes) para correção das taxas de incidência, que tendem a apresentar grande instabilidade, dado as pequenas populações dos bairros sob risco de adoecimento. Utiliza-se como parâmetro informações de áreas adjacentes de cada bairro para conter o efeito de flutuações aleatórias ${ }^{1}$. A matriz de vizinhança foi produzida de forma automatizada. A população sob risco do bairro e de sua vizinhança e foi realizada no software livre TerraView 3.1.4 (http://www.dpi.inpe.br/terraview/index.php).

Para a análise dos padrões espaciais de ocorrência da doença, calculou-se o Índice de Moran Local (LISA), a partir dos valores da correlação entre taxas para cada uma das unidades sob estudo, a uma significância de $0,05^{6}$.

No período avaliado, foram notificados 979 casos novos de TB em Vitória, perfazendo uma incidência média de 57,5/100mil habitantes. 0 município, que em 2000 contava com 292.304 habitantes distribuídos em uma área de $95 \mathrm{~km}^{2}$, encontra-se dividido em um total de 79 bairros, agrupados em sete Regiões Administrativas (RAs).

A despeito da pequena extensão territorial do município, estas exibem marcantes diferenças históricas, demográficas e socioeconômicas, sendo as regiões da Praia do Canto e Continental as que apresentam os melhores indicadores, ao passo que as populações de Santo Antônio e São Pedro possuem as condições de vida mais precárias ${ }^{7}$.

Nos relatórios rotineiramente produzidos pela Gerência de Vigilância em Saúde da Secretaria Municipal de Saúde, estas são as unidades de análise utilizadas. Destacam-se as diferenças entre a carga de doença nas RAs Centro $(85,7)$ e São Pedro $(84,7)$, com as maiores taxas, frente às Regiões Praia do Canto $(36,5)$ e Continente $(30,7)$, onde encontram-se as mais baixas. As demais RAs exibiram incidências semelhantes à do município (Figura 1).

Para análise da distribuição da TB por bairros, foram calculadas inicialmente as taxas médias de incidência brutas. A correção pelo LEBayes produziu importantes alterações nos valores, afetando praticamente todo o conjunto de dados. Os bairros com taxas brutas muito elevadas apresentaram

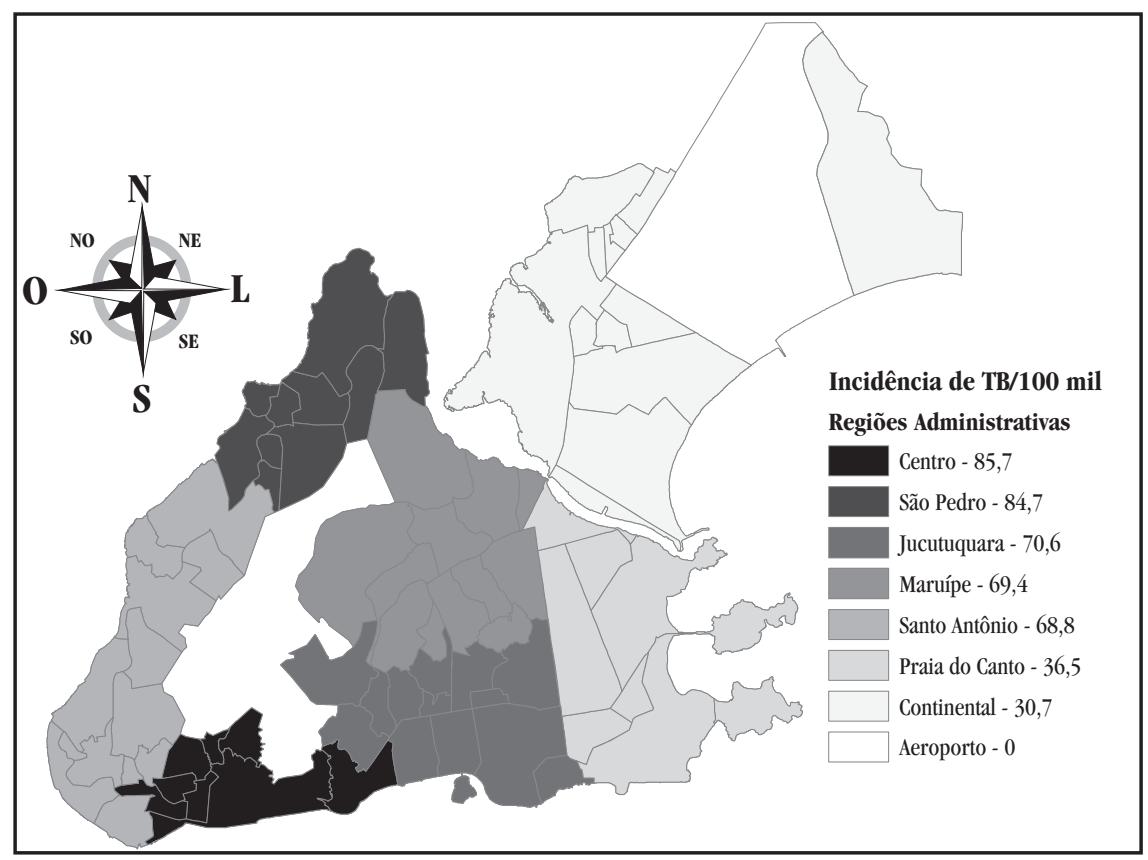

Figura 1 - Distribuição espacial da incidência de tuberculose no município de Vitória, por regiões administrativas, 2000-2005. 
importantes reduções após a correção, como Piedade $(163,7$ para 92,6), Vila Rubim $(197,2$ para 133,8) e São José $(174,9$ para 134,4) por $100 \mathrm{mil}$.

As estimativas de risco produzidas pelo LEBayes revelam áreas com elevado risco de adoecimento por TB, com bairros onde 0 valor da estimativa calculada alcança valores entre 130 a 160/100 mil, três vezes superiores à taxa de incidência do município. Em contrapartida, em diversos bairros a correção bayesiana foi inferior a 35/100 mil (Figura 2).

Por fim, procedeu-se a análise da existência de autocorrelação espacial entre as estimativas bayesianas do risco de adoecimento por TB dos bairros. Na Figura 3, são exibidos em destaque os bairros que, através do cálculo do LISA, apresentaram estimativas das taxas de incidência estatisticamente correlacionadas $(\mathrm{p}<0,05)$ com as existentes em seus vizinhos. Observando as taxas de incidência destes bairros, que são exibidas na Figura 2, classificou-se estas regiões como de alto e baixo risco, tomando como parâmetro a taxa média municpal. Desta forma, Jabour/Maria Ortiz/Segurança do Lar foi definida como de baixa endemicidade, a de Ariovaldo Favalessa/Bela Vista/ Santo Antônio como de risco intermediário, estando próxima à média municipal, e as de Comdusa/Grande Vitória/llha das Caieiras/ Santo André/Santos Reis e Bento Ferreira/Gurigica/Horto como de elevado risco para o adoecimento por TB.

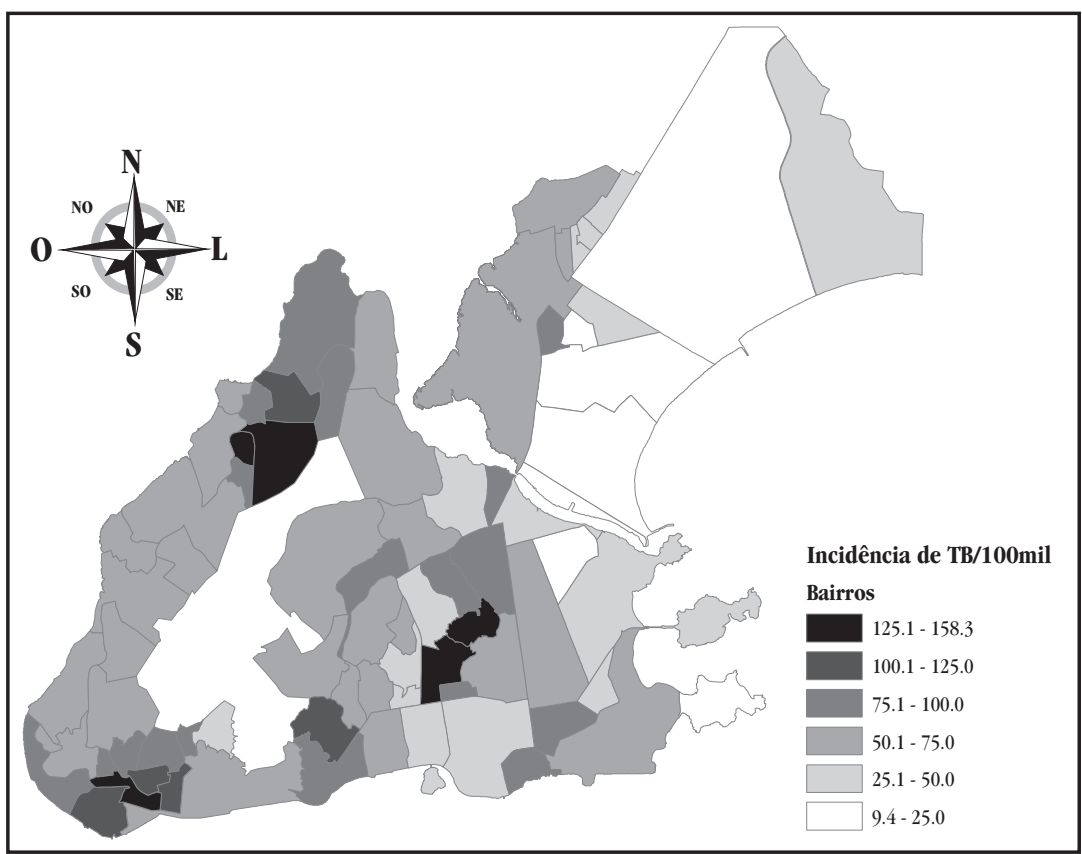

Figura 2 - Distribuição espacial da estimativa do risco de adoecimento (LEBayes) por tuberculose no município de Vitória, por bairros, 2000-2005.

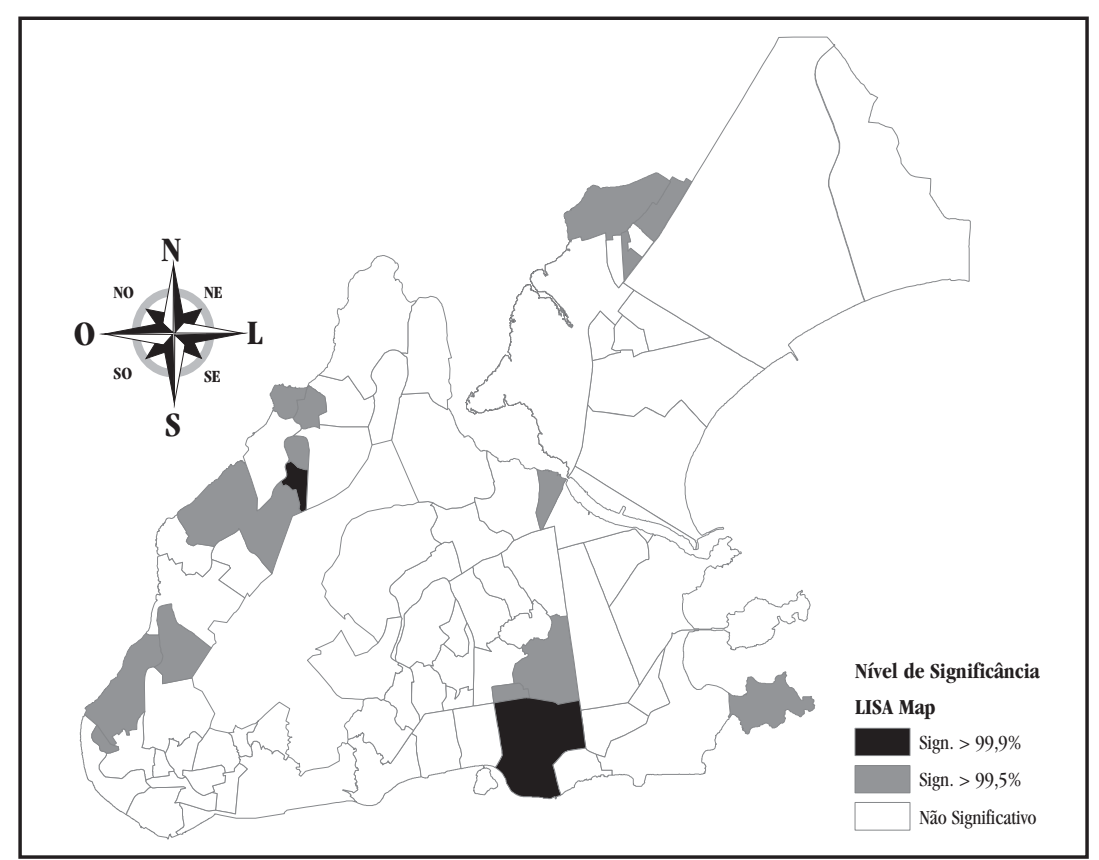

Figura 3 - Mapa da significância do Índice de Moran Local (LISA Map) utilizando-se as estimativa LEBayes para tuberculose no município de Vitória, por bairros, 2000-2005. 
0 principal achado deste estudo foi a intensa heterogeneidade com que a TB se distribui espacialmente no município de Vitória. Tal heterogeneidade tem sido demostrada em outras regiões ${ }^{3101214}$. A despeito da extensão de $95 \mathrm{~km}^{2}$ informada pelo IBGE, quando se excluem as áreas não habitadas do Parque Industrial, do Maciço Central e do Aeroporto, o município passa a contar com apenas $35,5 \mathrm{~km}^{2}$. É neste espaço que os cerca de 300 mil moradores e 160 casos anuais de TB se distribuem.

Deste fenômeno decorrem, ao menos, duas implicações. A primeira trata da estreita relação que a TB conserva com a categoria espaço e sua organização. Barcellos \& Bastos ${ }^{2}$, utilizando conceitos da Geografia para explicar a dualidade pertinente a esta categoria, mostram que, por um lado, o espaço é historicamente modelado pela ação da sociedade sobre a natureza ao passo que, neste processo, incorpora à sua conformação a estrutura e a dinâmica social.

Utilizando este referencial de espaço torna-se possível compreender, para além do nível individual, por que a TB, uma doença infecciosa transmitida pelo ar pessoa a pessoa, não atinja indistintamente todas as áreas de Vitória, mesmo que geograficamente tão próximas.

As taxas mais elevadas foram observadas nos bairros que apresentaram os piores indicadores de qualidade de vida urbana ${ }^{7}$. Este achado mostra concordante com a pesquisa de Souza e cols ${ }^{15}$ que, avaliando as possibilidades de implantação de uma sistema de vigilância baseado em informações geográficas e censitárias no município de Olinda, PE, demonstraram no nível ecológico uma importante associação entre baixo nível socioeconômico e a ocorrência de TB. Outros autores também destacam a capacidade desta metodologia de identificar áreas e populações prioritárias às ações de controle ${ }^{12} 18$.

0 trabalho de Barcellos \& Bastos ${ }^{2}$ trata da definição da unidade de análise, e como esta seleção influencia na percepção do fenômeno investigado. Assim, a análise dos mapas de risco produzidos pode resultar em interpretações discordantes, quando tomados como unidade de análise as RAs ou os bairros. Na primeira possibilidade, parte-se do pressuposto que os bairros que formam uma RA apresentam características tais que permitam uma distribuição homogênea dos casos em seu interior, como apresentado na Figura 1. Contudo, como demonstraram Souza e cols ${ }^{16}$, a TB é um fenômeno que tende a apresentar grandes variações entre pequenas áreas. Os autores mostram que, na definição desta estrutura espacial, somam-se aos fatores socioeconômicos outras variáveis relacionadas com aspectos individuais e familiares associados ao surgimento de novos casos. Em nosso estudo encontramos que quatro dos nove bairros com as maiores estimativas de risco não se encontravam nas RAs de maior incidência. Dessa forma, nosso estudo corrobora com a proposição de que a avaliação da TB no espaço deva tomar como unidade de análise os menores territórios para os quais existam dados, a exemplo dos setores censitários.

Assim sendo, os dados produzidos pelo cálculo do Índice de Moran Local (LISA) somam-se ao conjunto de técnicas da vigilância em saúde na seleção de áreas de intervenção. Destacase como limitação do uso das taxas de incidência corrigidas pelo método LEBayes a tendência deste induzir a autocorrelação espacial, podendo gerar artefatos, como regiões formadas por bairros com pequeno número de casos e alta incidência, no quais a correção do risco se aproxima da média de seus vizinhos com elevadas endemicidades e grandes populações ${ }^{11}$.

A segunda implicação destes achados relaciona-se com as possibilidades metodológicas de controle da endemia. A observação de que a TB se distribua de forma tão heterogênea, concentrando-se em pequenas áreas ou bairros, inviabiliza a utilização das RAs como suporte às ações de vigilância em saúde. Ainda que, a princípio isto gere a necessidade de um maior esforço na qualificação dos dados obtidos e registrados nos sistemas de informação de saúde, este padrão de distribuição permite a estruturação da uma vigilância mais focada e, portanto, eficiente.

\section{REFERÊNCIAS}

1. Assunção RM, Barreto SM, Guerra HL, Sakurai E. Mapas de taxas epidemiológicas: Uma abordagem Bayesiana. Cadernos de Saúde Pública 14: 713-723, 1998.

2. Barcellos C, Bastos FI. Geoprocessamento, ambiente e saúde: uma união possível? Cadernos de Saúde Pública 12: 389-397, 1996.

3. Bishai WR, Greham NM, Harrington S, Pope, DS, Hooper, N, Astemborski J, Sheely L, Vlahov D, Glass GE, Chaisson RE. Molecular and geographic patterns of tuberculosis transmission after 15 years of directly observed therapy. Journal of American Medical Association 280: 1679-1684, 1998.

4. Carvalho MS, Souza-Santos R. Análise de dados espaciais em saúde pública: métodos, problemas, perspectivas. Cadernos de Saúde Pública 21: 361-378, 2005

5. Costa JSD, Gonçalves H, Menezes AMB, Devens E, Piva M, Gomes M, Vaz M. Controle epidemiológico da tuberculose na cidade de Pelotas, Rio Grande do Sul, Brasil: adesão ao tratamento. Cadernos de Saúde Pública 14: 409-415, 1998.

6. Cressie NAC. Statistics for Spatial Data. Wiley-Interscience, Belmonte, 1993.

7. Maciel ELN. Sistema de Informação em Geoprocessamento de Mortalidade por Causas Externas - SIGMOR. Espírito Santo, 2007. Relatório Final do Projeto de Pesquisa apresentado ao Conselho Nacional de Desenvolvimento Científico e Tecnológico, 2007.

8. Ministério da Saúde. Saúde Brasil 2005: uma análise da situação de saúde no Brasil. Secretaria de Vigilância em Saúde, Departamento de Análise de Situação em Saúde. $2^{\text {a }}$ edição. Brasília, 2005.

9. Ministério da Saúde. Relatório de Situação - Espírito Santo. Sistema Nacional de Vigilância em Saúde. $2^{a}$ edição. Brasília, 2006.

10. Moonan PK, Bayona M, Quitugua TN, Oppong J, Dunbar D, Jost Jr KC, Burgess G, Singh KP, Weis SE. Using GIS technology to identify areas of tuberculosis transmission and incidence. International Journal of Health Geographics 3: 23-32, 2004.

11. Morais-Neto OL, Barros MB, Martelli CM, Silva SA, Cavenaghi SM, Siqueira Jr JB. Diferenças no padrão de ocorrência da mortalidade neonatal e pós-neonatal no Município de Goiânia, Brasil, 1992-1996: análise espacial para identificação das áreas de risco. Cadernos de Saúde Pública 17: 1241-1250, 2001.

12. Munch Z, Van Lill SW, Booysen CN, Zietsman HL, Enarson DA, Beyers N. Tuberculosis transmission patterns in a high-incidence area: a spatial analysis. The International Journal of Tuberculosis and Lung Disease 7: 271-277, 2003.

13. Ruffino-Netto A. Tuberculose: a calamidade negligenciada. Revista da Sociedade Brasileira de Medicina Tropical 35: 51-58, 2002.

14. Serpa IM, Pardo CL, Hernandez RA. Un estudio ecológico sobre tuberculosis en un municipio de Cuba. Cadernos de Saúde Pública 19: 1305-1312, 2003. 
15. Souza WV, Albuquerque MFM, Barcellos CC, Ximenes RA, Carvalho MS. Tuberculose no Brasil: construção de um sistema de vigilância de base territorial. Revista de Saúde Pública 39: 82-89, 2005.

16. Souza WV, Ximenes R, Albuquerque MFM, Lapa TM, Portugual JL, Lima MLC, Martelli CMT. The use of socioeconomic factors in mapping tuberculosis risk areas in a city of northeastern Brazil. Panamerican Journal of Public Health 8: 403-410, 2000.
17. World Health Organization. Global Tuberculosis Control - Surveillance, Planning, Financing. WHO Report 2006. Geneva, 2006.

18. Xavier MIM, Barreto ML. Tuberculose na Cidade de Salvador, Bahia, Brasil: o perfil na década de 1990. Cadernos de Saúde Pública 23: 445-453, 2007. 